\title{
Telemedizin
}

\section{Das bringen die neuen EBM-Positionen zur Videosprechstunde}

\author{
Eine Technikpauschale, die außerhalb der Gesamtvergütung \\ läuft, und eine Video-Kontakt-Ziffer: Die neuen Leistungen \\ im EBM für die Videosprechstunde klingen vielversprechend. \\ Am Fernbehandlungsverbot rütteln sie aber nicht.
}

A b April können Vertragsärzte Videosprechstunden erbringen und auch mit der Kassenärztlichen Vereinigung (KV) abrechnen. Anders als die Förderung des elektronischen Arztbriefes mit 28 Cent (GOP 86900) für den Sender und 27 Cent (GOP 86901) für den Empfänger, kann sich der Zuschlag für die Videokonsultation auch sehen lassen.

\section{Die Technik zahlt der Arzt}

Auf zwei neue Gebührenordnungspositionen (GOP) haben sich KBV und GKV-Spitzenverband geeinigt. Für die Bereitstellung der notwendigen Hardund Software gibt es die GOP 01450 (40 Punkte, 4,21 Euro). Dieser Technikzuschlag ist mehr als berechtigt, da es die Ärzte sind, die sich um einen sicheren Übertragungsweg für die Videosprechstunde kümmern müssen. Bereits im vergangenen Herbst hatten KBV und Kassen die Anforderungen an die Technik in eine Ergänzung zum Bundesmantelvertrag Ärzte (Anlage 31 b) gepackt.

Demnach ist ein Videodienst mit einer Ende-zu-Ende-Verschlüsselung erforderlich, dabei dürfen die Server des Anbieters nur innerhalb der EU stehen, und der Anbieter darf keine Patienten- oder Videodaten langfristig speichern. Patienten müssen sich zudem ohne Account anmelden können, wobei der Arzt den Kontakt initiieren muss und der Zugang auf maximal einen Monat befristet sein muss. Die Praxis muss sich von dem jeweiligen Anbieter bestätigen lassen, dass er sich an diese Spielregeln hält und dies gegenüber der KV nachweisen. Anders als die Praxis-EDV-Anbieter müssen sich die Videodienst-Anbieter bislang aber nicht von der KBV zertifizieren lassen. Patienten hingegen benötigen nicht mehr als einen Rechner mit Internetzugang, Webcam und Mikrofon.

4,21 Euro je Videosprechstunde sind zwar nicht üppig. Der Bewertungsausschuss gehe aber davon aus, dass sich die Technik bereits bei zwei Videosprechstunden pro Woche rechne, so die KBV. Laut Standesvertretung liegen die Lizenzgebühren für Videodienste aktuell bei etwa 100 Euro im Quartal. Und mit den neuen Ziffern könnten Ärzte bis zu 800 Euro Förderung pro Jahr einstreichen.

Tatsächlich ist der Technikzuschlag, der außerhalb der morbiditätsorientierten Gesamtvergütung finanziert wird, mit einem Punktzahlvolumen je Arzt belegt. Das heißt, pro Arzt werden maximal 1.899 Punkte pro Quartal vergütet, das entspricht 47,5 Videosprechstunden oder beim derzeitigen Orientierungswert im EBM (dem Preis für einen EBM-Punkt) von 10,53 Cent rund 200 Euro pro Quartal. Für alle weiteren Videosprechstunden gibt es den Technikzuschlag nicht.

Die Förderung hat aber noch einen Haken. Der tatsächliche Videokontakt ist laut Bewertungsausschuss - wie auch der Telefonkontakt - eine Substitutionsleistung für den Arztkontakt vor Ort in der Praxis. Daher ist er prinzipiell auch mit der Versicherten- oder Grundpauschale abgedeckt. Auch im Bereitschaftsdienst oder ärztlichen Notdienst darf die neue Position nicht angesetzt werden.

\section{Nicht für den Erstkontakt}

Nur in Quartalen, in denen der Patient nicht auch noch die Praxis aufsucht, kann daher die GOP 01439 für den Videokontakt abgerechnet werden (88 Punkte, 9,27 Euro). Die beiden neuen Positionen gelten allerdings nur für die Folgebegutachtung. Es muss also zuvor ein persönlicher Arzt-Patienten-Kontakt in derselben Praxis stattgefunden haben. Für die GOP 01439 muss dieser in einem der beiden Vorquartale erfolgt sein. Dies sei notwendig, so die KBV, damit die Ärzte nicht gegen das Fernbehandlungsverbot verstoßen. Außerdem werden beide Leistungen zunächst nur für sechs Indikationen vergütet, wovon drei für Dermatologen Relevanz haben:

-visuelle postoperative Verlaufskontrolle einer Operationswunde,

- visuelle Verlaufskontrolle einer/von akuten, chronischen und/oder offenen Wunde(n),

-visuelle Verlaufskontrolle einer/von Dermatose(n), auch nach strahlentherapeutischer Behandlung.

\section{Schriftliche Einwilligung Pflicht}

Zusätzlich wurde für eine Reihe von EBM-Ziffern, die mindestens drei persönliche Arzt-Patienten-Kontakte im Behandlungsfall voraussetzen, vereinbart, dass einer dieser persönlichen Kontakte auch im Rahmen einer Videosprechstunde stattfinden kann. Dies gilt für die GOP 02310, 07310, 07311, 07330, 07340, 10330, 18310, 18311, 18330 und 18340.

Ärzte müssen sich vor der Videosprechstunde eine schriftliche Einwilligung beim Patienten für den neuen Datenerhebungs- und -übermittlungsweg einholen, dies zählt zu den obligaten Leistungsinhalten der GOP 01439 und 01450. Neben Hautärzten dürfen die Videosprechstunde bislang 15 weitere Fachgruppen abrechnen. Rebekka Höhl 